BUll. austral. Math. SOC.

06E99, 06B10

VOL. 32 (1985), 177-193.

\title{
SOME PROPERTIES OF THE LATTICE OF \\ SUBALGEBRAS OF A BOOLEAN ALGEBRA
}

\author{
Ivo DüNTSCH
}

We investigate the structure of the lattice of subalgebras of an infinite Boolean algebra; in particular, we make a contribution to the question as to when such a lattice is simple.

\section{Introduction}

For a Boolean algebra $\left(D,+,{ }^{\circ},-, 0,1\right)$, the set sub $D$ of all subalgebras is an algebraic lattice under set inclusion with least element $2=\{0,1\}$ and greatest element $D$. If $A, B \leq D$, then $A \wedge B$ is just $A \cap B$, and $A \vee B$ is the subalgebra of $D$ generated by $A \cup B$.

One of the earliest results in the study of $\mathrm{sub} D$ is the fact that, if $D$ is finite, then $s u b D$ is dually isomorphic to a finite partition. lattice, the base set being $A t(D)$, the set of all atoms of $D$, see [1]. Subsequently it was shown by $D$. Sachs that, for an arbitrary Boolean algebra $D$, sub $D$ is dually isomorphic to a sublattice of a partition lattice, and that sub $D$ characterizes $D$. Birkhoff's result cited above implies that sub $D$ is simple, if $D$ is finite.

In this note, the structure of sub $D$ is investigated further; in particular we make a contribution to the question when sub $D$ is simple for

Received 7 January 1985

Copyright Clearance Centre, Inc. Serial-fee code: 0004-9727/85 $\$ A 2.00+0.00$. 
infinite $D$.

\section{Notation}

For any lattice $L$, we set $[x, y)=\{z \in L \mid x \leq z<y\}$, $[x]=\{z \in L \mid x \leq z\} ;$ other intervals are defined analogously.

Let $D$ be a Boolean algebra; for $M \subseteq D$ we define $M^{+}=\{x \in M \mid x>0\},-M=\{\vec{x} \mid x \in M\}$, and $[M]$ to be the subalgebra of $D$ generated by $M$. If $M=\{x\}$, we just write $[x]$ instead of $[\{x\}]$. For $A \leq D, x \in D \backslash A$, we call $[A \cup[x\}]$ a simple extension of $A$, and denote it by $A(x)$. Note that every element of $A(x)$ is of the form $u \circ x+v \circ \bar{x}$ for some $u, v \in A$.

For $d \in D^{+}, D \mid d=\{x \in D \mid x \leq d\}$ is the relative algebra of $d$ in $D$. Note that $D \mid d$ also is the principal ideal of $D$ generated by $d$, and we sometimes alternatively write (d] for $D / d$, if we want to emphasize this fact. It is well known that $D$ is isomorphic to $D|d \times D| \bar{d}$; conversely, if $D$ is isomorphic to $A \times B$, then there exists a $d \in D$ such that $A \cong D \mid d$, and $B \cong D \mid \bar{d}$.

If $C$ is a linearly ordered set with least element, the set of all finite unions of right closed, left open intervals is a Boolean subalgebra of the power set of $C$, and denoted by $I(C)$; this algebra is called the interval algebra of $C$. In an unpublished paper, s. Todorčevic [6] has shown that for an interval algebra $D$, sub $D$ is sectionally complemented, that is Sub $A$ is complemented for every $A \leq D$.

For the remaining unexplained notation and terminology the reader is referred to Grätzer's book [3].

\section{General structure of Sub D}

A lattice $L$ is called semimodular if, for $x, y \in L$, the fact that $x$ covers $x \wedge y$ implies that $x \vee y$ covers $y$. Note that every modular lattice is semimodular.

PROPOSITION 2.1. If $|D|=8$, then sub $D$ is modular; if $|D| \geq 16$, then sub $D$ is not semimoduzar.

Proof. If $|D|=8$, then sub $D$ is easily seen to be a diamond, so it is modular. If $|D| \geq 16$, then $D$ has a subalgebra with four atoms, 
so, let without loss of generality $D$ be generated by its atoms $\{a, b, c, d\}$, and set $A=[a+c], B=[c+d]$. Then $A$ covers $A \cap B=2$, but $A \vee B=D$ does not cover $B$.

In contrast to this, Sachs [5] has remarked that (Sub D) ${ }^{d}$, the dual lattice of $\operatorname{sub} D$, is semimodular; however, (Sub $D)^{d}$ usually is not algebraic.

PROPOSITION 2.2. If $D$ is infinite, then $(\mathrm{Sub} D)^{d}$ is not algebraic.

Proof. We show that no dual atom of sub $D$ is dually compact. As noted by sachs, the dual atoms of sub $D$ are of the form $I U-I$, where $I$ is the intersection of two different prime ideals $P_{1}$ and $P_{2}$ of $D$, so, let $A \leq D$ have this form.

Assume that $\{a, b, c\} \subseteq D \backslash A$, and $a, b, c$ are pairwise disjoint. since $a \circ b=0$, we suppose, without loss of generality, that $a \in P_{1} \backslash P_{2}$; then $a \circ c=0$ implies $c \in P_{2} \backslash P_{1}$, and from $a \circ b=c \circ b=0$ we get $b \in P_{1} \cap P_{2} \leq A$, a contradiction. Now we choose an element $u$ from $D \backslash A$, and a set $\left\{u_{i} \mid i<\omega\right\}$ of pairwise disjoint elements such that $u_{0}=u$, and $u_{i} \in I$ for $0<i<\omega$. For each $i<\omega$, let $m_{i}=u_{0}+\ldots+u_{i}$, and, for $j<\omega$, let $M_{j} \leq D$ be generated by $\left\{m_{i} \mid j \leq i<\omega\right\}$; then, $\left\{M_{j} \mid j<\omega\right\}$ is a decreasing chain of subalgebras of $D$ with $\cap\left\{M_{j} \mid j<\omega\right\}=2 \leq A$. Since no $m_{i}$ is in $A$, we have $M_{j} \ddagger A$ for all $j<\omega$, which implies that $A$ is not dually compact.

The next result shows that $s u b D$ is in fact far from being distributive. Call an element $a$ of a lattice $L$

(i) distributive, if $a \vee(x \wedge y)=(a \vee x) \wedge(a \vee y)$ for all $x, y \in L$

(ii) prime, if $x \wedge y \leq a$ implies $x \leq a$ or $y \leq a$

(iii) irreducible, if $x \wedge y=a$ implies $x=a$ or $y=a$. If $a$ is prime, it is irreducible, and if $L$ is distributive, the converse also holds. 
PROPOSITION 2.3.

1. Sub $D$ has no proper distributive or prime elements.

2. $A \leq D$ is irreducible if and only if $A$ is a dual atom of Sub $D$.

\section{Proof.}

1. We may suppose that $|D| \geq 8$; let $2<A<D, b \in D \backslash A$, and $c \in A(b) \backslash A, c \neq b$. Then $A=A \vee([b] \cap[c])$, but $c \in A(c) \cap A(b)$, showing that $A$ is not distributive. since $[c] \cap[b]=2 \leq A$, and $[c],[b] \$ A, A$ is not prime.

2. The if-part is obvious, so, let $A<D$ be irreducible. First, assume that $A$ is not of the form $I U-I$ for some ideal $I$ of $D$. Then there exist $a \in A, b_{1}, b_{2} \in D \backslash A$, such that $b_{1} \circ \bar{a}=b_{2} \circ a=0$. Let $x \in A\left(b_{1}\right) \cap A\left(b_{2}\right)$; then there exist $s_{1}, s_{2}, t_{1}, t_{2} \in A$, such that $x=s_{1} \circ b_{1}+s_{2} \circ \overline{b_{1}}=t_{1} \circ b_{2}+t_{2} \circ \overline{b_{2}}$. So, $a \circ x=a \circ t_{2} \circ \overline{b_{2}}$ $=a \circ t_{2} \in A$, and $\bar{a} \circ x=\bar{a} \circ s_{2} \circ \overline{b_{1}}=\bar{a} \circ s_{2} \in A$, which together imply that $x \in A$; it follows that $A=A\left(b_{1}\right) \cap A\left(b_{2}\right)$, contradicting the fact that $A$ is irreducible. Thus, let $A=I U-I$ for some ideal $I$ of $D$, and assume that $I$ is not the intersection of two prime ideals; then there exists a $B \leq D$ such that $A \cap B=2$, and $B$ is generated by its atoms $b_{1}, b_{2}, b_{3}$. If $x \in A\left(b_{1}\right) \cap A\left(b_{2}\right) \cap A\left(b_{3}\right)$, then there exist $r_{i}, s_{i}, t_{i} \in \dot{A}, 1 \leq i \leq 2$, such that

$$
\begin{aligned}
x & =r_{1} \circ b_{1}+r_{2} \circ b_{2}+r_{2} \circ b_{3} \in A\left(b_{1}\right) \\
& =s_{1} \circ b_{2}+s_{2} \circ b_{3}+s_{2} \circ b_{1} \in A\left(b_{2}\right) \\
& =t_{1} \circ b_{3}+t_{2} \circ b_{1}+t_{2} \circ b_{2} \in A\left(b_{3}\right) .
\end{aligned}
$$

Using $A=I \cup-I$, it is straightforward, if somewhat cumbersome, to show that $x \in A$, again contradicting the irreducibility of $A$.

The question of the existence of prime ideals in sub $D$ will be touched on later.

\section{Congruences on Sub D}

We start with the following easy observation: 
LEMMA 3.1. Let $\theta$ be a nontrivial congmence on sub $D$; then $A \equiv 2(\theta)$ for every finite $A \leq D$.

Proof. It is enough to show that $[u] \equiv 2(\theta)$ for every $u \in D$. Since $\theta$ is nontrivial, there exist $A, B \leq D$, such that $A \subseteq B$, $A \neq B$, and $A \equiv B(\theta)$. Let $b \in B \backslash A$; then

$$
2=[b] \cap A \equiv_{\theta}[b] \cap B=[b] \text {. }
$$

If $u \in D \backslash[b]$, let $C$ be generated by $\{b \circ u, \bar{b} \circ \bar{u}\}$; then

$$
2=[u] \cap C \equiv_{\theta}[u] \cap C(b)=[u] \text {. }
$$

COROLLARY 3.2. Sub $D$ is subdirectly irreducible, weakly modular, and weakly complemented.

In the sequel, we shall just write $A \equiv B$, if $A$ is congruent to $B$ modulo the smallest nontrivial congruence on sub $D$. Note that 3.2 implies that, if $A \leq D$ and sub $A$ is simple, then $A \equiv 2$; consequently, if $D=D_{0} \times D_{1} \times \ldots \times D_{n}$, and sub $D_{i}$ is simple for each $i \leq n$, then Sub $D$ is simple.

Next, we want to give some simple conditions for sub $D$ to be simple.

PROPOSITION 3.3. If $D=A \times A$, then sub $D$ is simple.

Proof. Let $E=\{(a, a) \mid a \in A\} \leq D, u=(0,1) \in D, B=A \times 2$, $C=2 \times A$; then $E(U)=D$, and $B \cap E=C \cap E=2$, implying $B, C \equiv 2$. on the other hand, $D=B \vee C$, thus, $D \equiv 2$.

In particular, every homogeneous $D$ has sub $D$ simple.

PROPOSITION 3.4. If $|D|=\lambda \geq \omega$, and $D$ contains a free subalgebra with $\lambda$ generators, then sub $D$ is simple.

Proof. Choose some $u \in D$, such that both $(u]$ and $(\bar{u}]$ contain an independent set of cardinality $\lambda$. Let $\left\{m_{i} \mid i<\lambda\right\}$ be an enumeration of $(u]^{+}$, $\left\{b_{i} \mid i<\lambda\right\}$ an independent set of elements below $\bar{u}$, and let $F \leq D$ be generated by this set; furthermore, let $B_{1} \leq D$ be generated by $(u]$, that is $B=(u] \cup[\bar{u})$. For each $i<\lambda$ set $c_{i}=m_{i}+b_{i}$, and let $C \leq D$ be generated by the $c_{i}$. The independence of the $b_{i}$ then implies that $B_{1} \cap C=2$. Also, $m_{i}=c_{i} \circ \bar{b}_{i}$ and $b_{i}=c_{i} \circ \bar{m}_{i}$, so we have 
$B_{1} \vee F=B_{1} \vee C=F \vee C$. Since $F$ is free, $F \equiv 2$ by the preceding proposition, and therefore $C \equiv F \vee C$. Hence, $2=B_{1} \cap C \equiv B_{1} \cap(F \vee C)=B_{1} \cap\left(B_{1} \vee C\right)=B_{1}$. By symetry, we find that $B_{2}=(\bar{u}] \cup[u)$ also is congruent to 2 . Since $D=B_{1} \vee B_{2}$, sub $D$ is simple.

In particular, if $D$ is complete, sub $D$ is simple by the theorem of Balćar and Frariek.

If $A \leq D, u \in D$, call $u$ independent of $A$, if $a \circ u>0$ and $a \circ \bar{u}>0$ for all $a \in A^{+}$. Note that this is equivalent to $A \cap(u]=A \cap(\bar{u}]=\{0\}$.

PROPOSITION 3.5. If $D$ is the free product of $A$ and $B$, then Sub $D$ is simple.

Proof. This follows from the simple fact, that $C \leq D, u \in D$ independent of $C$, imply $C \equiv 2$ : Indeed, independence implies that for $E_{1}=(u] \cup[\bar{u}), E_{2}=(\bar{u}] \cup[u)$, we have $C \cap E_{1}=C \cap E_{2} \equiv 2$; since $C \equiv C(u)$, this gives us $C(u) \cap E_{1} \equiv 2$ and $C(u) \cap E_{2}=2$. On the other hand, $C \leq\left(C(u) \cap E_{1}\right) \vee\left(C(u) \cap E_{2}\right)$. If $c \in C$, then $c \circ u \in C(u) \cap E_{1}$, and $c \circ \bar{u} \in C(u) \cap E_{2}$. This shows $C \equiv 2$. For the rest, observe that each $b \in B \backslash 2$ is independent of $A$ and vice versa.

Now, let us turn to conditions which ensure us that sub $D$ is not simple. Each ideal $I$ of sub $D$ induces an equivalence relation $\theta_{I}$ on sub $D$, if we let $A \equiv B\left(\theta_{I}\right)$ if there exists a $C \in I$ such that $A \vee C=B \vee C$. Clearly, $\theta_{I}$ is a v-congruence on sub $D$. If $I$ is a distributive element of the lattice of ideals of Sub $D$, then ${ }^{\theta_{I}}$ is a lattice congruence on Sub $D$, see [3] III.3.4. For each cardinal $\gamma$, $\omega \leq \gamma \leq \lambda=|D|$, let $I_{\gamma}=\{A \leq D|| A \mid<\gamma\}$, and $\theta_{\gamma}$ be the relation defined above. The following lemma simplifies later considerations.

LEMMA 3.6. Let $|D|=\lambda$; for $\omega \leq \gamma \leq \lambda,{ }_{\gamma}$ is a congruence if and only if the following condition holds:

If $A, B, C \leq D,|C|<\gamma$, and $B \leq A \vee C$, then there exists on 
$S \in I_{\gamma},(A \cap B) \vee S=B \vee S$. Moreover, if $\gamma$ is regular, $C$ can be assumed to have only four elements.

Proof. We only show sufficiency, and it is enough to prove that $A \equiv B\left(\theta_{\gamma}\right)$ and $Q \leq D$ imply $Q \cap A \equiv Q \cap B\left(\theta_{\gamma}\right)$. Let, for some $C \in I_{\gamma}$, $A \vee C=B \vee C$, and set $Q_{1}=Q \cap(A \vee C)=Q \cap(B \vee C)$; then $Q_{1} \cap A=Q \cap A$, and $Q_{1} \cap B=Q \cap B$, so we can suppose, without loss of generality, that $Q \leq A \vee C=B \vee C$. By the condition, there exist $S_{1}$, $S_{2} \in I_{\gamma}$, such that $(Q \cap A) \vee S_{1}=Q \vee S_{1}$, and $(Q \cap B) \vee S_{2}=Q \vee S_{2}$; hence, $(Q \cap A) \vee S_{1} \vee S_{2}=(Q \cap B) \vee S_{1} \vee S_{2}$, and $S_{1} \vee S_{2} \in I_{\gamma}$.

For the second part, let $C=\left\{c_{i} \mid i<\delta\right\}, \delta<\gamma$, and $Q \leq A \vee C$. Set $A_{0}=A, A_{\alpha+1}=A_{\alpha}\left(c_{\alpha}\right)$, and $A_{\alpha}=U\left\{A_{\beta} \mid \beta<\alpha\right\}$, if $\alpha$ is a limit. Then $A \vee C=U\left\{A_{\alpha} \mid \alpha<\delta\right\}$. For $i<\delta$, set $Q_{i}=Q \cap A_{i} ;$ then, $Q=U\left\{Q_{i} \mid i<\delta\right\}$. It suffices to show that for each $i<\delta$ there exists an $S_{i} \in I_{\gamma}$, such that $Q_{i} \vee S_{i}=(Q \cap A) \vee S_{i}$. Set $S=\left[\cup\left\{S_{i} \mid i<\delta\right\}\right]$; then $Q \vee S=(Q \cap A) \vee S$, and $S \in I_{\gamma}$ by the regularity of $\gamma$. Let $i=0$; then $Q_{0}=Q \cap A_{0}=Q \cap A$, and we set $S_{O}=2$. Suppose that for all $\alpha<\beta<\delta$ we have $Q_{\alpha} \vee S_{\alpha}=(Q \cap A) \vee S_{\alpha}, S_{\alpha} \in I_{\gamma}$. If $\beta$ is a limit, set $S_{\beta}=\left[\cup\left\{S_{\alpha} \mid \alpha<\beta\right\}\right]$, and note that $Q_{\beta}=\cup\left\{Q_{\alpha} \mid \alpha<\beta\right\}$. So, let $\beta=\alpha+1$; then, $Q_{\beta}=Q \cap A_{\alpha+1}=Q \cap A_{\alpha}\left(c_{\alpha}\right) ;$ thus, by our hypothesis, there exists a $T \in I_{\gamma}$ which satisfies $\left(Q \cap A_{\alpha}\right) \vee T=\left(Q \cap A_{\alpha}\left(c_{\alpha}\right)\right) \vee T$. By the induction hypothesis there exists an $S_{\alpha} \in I_{\gamma}$ satisfying $\left(Q \cap A_{\alpha}\right) \vee S_{\alpha}=(Q \cap A) \vee S_{\alpha} ;$ now set $S_{\beta}=S_{\alpha} \vee T$.

The proof of the following easy lemma is left to the reader.

LEMMA 3.7. Let $D$ be infinite and sub $D$ be sectionally complemented; if $\omega \leq \gamma \leq|D|$, then $\theta_{\gamma}$ is a congmence if and only if the following condition holds:

If $C \in I_{Y}$, then every $Q \leq A \vee C$ disjoint from $A$ has cardinality Zess than $\gamma$. 
Our next aim is to describe the congruence lattice of sub $D$ for $D=F C(\alpha)$, the finite-cofinite algebra with $\alpha$ atoms. Note that $F C(\alpha)$ is a subalgebra of an interval algebra, hence its lattice of subalgebras is sectionally complemented.

PROPOSITION 3.8. Let $D=F C(\alpha)$, where $\alpha=\aleph_{\gamma}$. Then the congruences of sub $D$ form a chain of type $\gamma+3$, if $\gamma<\omega$, and of type $\gamma+2$ otheruise.

Proof. We first show that for all $\beta, \omega \leq \beta \leq \alpha$, the ideal $I_{\beta}$ has the property of 3.7 ; so, together with the two improper congruences, the $\theta_{\beta}$ form a chain of the desired type. Afterwards we proceed to show that every proper congruence on $s u b D$ is of the form $\theta_{\beta}$ for some infinite $\beta \leq \alpha$.

Let $C \in I_{\beta}$ and assume the existence of $A, B \leq D$, such that $B \leq A \vee C,|B|=\beta$, and $A \cap B=2$. Let $A t(B)=\left\{b_{i} \mid i<\beta\right\}$ be the set of atoms of $B$, and suppose $b_{i}=a_{1}^{i} \circ c_{1}^{i}+\ldots+a_{\tau(i)}^{i} \circ c_{\tau(i)}^{i}$, where $a_{j}^{i} \in A$ and $c_{j}^{i} \in C$. If $c_{j}^{i}$ is cofinite, then $a \circ c_{j}^{i} \notin A$ for only finitely many atoms of $A$. Since there are only less than $\beta$ elements $c_{j}^{i}$, we may suppose, without loss of generality, that each $b_{i}$ has the form $b_{i}=a_{1}^{i}+a_{2}^{i} \circ c_{2}^{i}+\ldots+a_{s(i)}^{i} \circ c_{s(i)}^{i}$, where each $c_{j}^{i}$ is finite. Now we set $s_{i}=a_{2}^{i} \circ c_{2}^{i}+\ldots+a_{\tau(i)}^{i} \circ c_{\tau(i)}^{i} ;$ since, for each $i<\beta, b_{i}$ is not an element of $A$, we must have $s_{i}>0$. On the other hand, each $c_{k}^{i}$ is finite, and there are only less than $\beta$ such $c_{k}^{i}$, so, we must have $s_{i}=s_{j}$ for some $i, j<\beta$. This contradicts $b_{i} \circ b_{j}=0$. Next, let $\psi$ be a nontrivial congruence on $\operatorname{sub} D$, and consider the property

(*) If $A \leq D,|A|=\lambda \geq \omega$, and $A \equiv 2(\psi)$, then $B \equiv 2(\psi)$ for all $B \in I_{\lambda^{+}}$.

We show this by induction. Call an atom $m$ of $A$ proper, if $m$ is an atom of $D$; otherwise call $m$ improper.

(a) Let $|A|=\omega$, and suppose that $A$ has infinitely many proper atoms 
$\left\{m_{i} \mid i<\omega\right\}$; since $A \equiv 2(\psi)$, we may assume that $A$ is generated by these atoms. Let $|B|=\omega$; then all atoms of $B$ are finite. Let $\left\{c_{i} \mid i<\omega\right\}$ be the set of atoms of $D$ which are $\leq$ some atom of $B$, but not in $A$, and let $C$ be generated by the $c_{i}$. If $C$ is finite, then $B=A \vee Q$ for some finite $Q \leq D$, and thus $B \equiv 2(\psi)$. Thus, let $c_{i} \neq c_{j}$ for $i \neq j$. clearly, $A \cap C=2$ and $A \vee B \leq A \vee C$. For each $i<\omega$, let $q_{i}=m_{i}+c_{i}$, and let $Q$ be generated by the $q_{i}$. Then, $Q \cap A=Q \cap C=2$, and $Q \vee A=Q \vee C=A \vee C$. This implies $Q \equiv C(\psi)$ and it follows that $C \equiv 2(\psi)$, observing that $Q \cap C=2$. Since $B \leq A \vee C$, we have $B \equiv 2(\psi)$.

(b) Let $|A|=\omega$, and $A$ be generated by the improper atoms $\left\{m_{i} \mid i<\omega\right\}$ For each $i<\omega$, let $m_{i}=x_{i}+y_{i}$, where $x_{i}$ is an atom of $D$, and $y_{i}=\vec{x}_{i} \circ m_{i}$. Let $Q$ be generated by the $x_{i}$, and $R$ be generated by the $y_{i}$; then, as before, $A \cap Q=A \cap R=R \cap Q=2$, and $A \vee Q=A \vee R=Q \vee R$. This implies $Q \equiv 2(\psi)$, and we can proceed as in (a) with $Q$ instead of $A$, noting that all atoms of $Q$ are proper.

Now suppose that (*) holds for all $\kappa<\lambda \leq \alpha$, and let $A, B \leq D$ such that $|A|=|B|=\lambda$, and $A \equiv 2(\psi)$.

(c) $A$ is generated by the $\lambda$ proper atoms $\left\{m_{i} \mid i<\lambda\right\}$. Let $\left\{c_{i} \mid i<\delta\right\}$ be the set of all atoms of $D$ which are below some atom of $B$, but not in $A$, and let $C$ be generated by the $c_{i}$. If $\delta<\lambda$, then $C \equiv 2(\psi)$ by our induction hypothesis, and thus $B \equiv 2(\psi)$, since $B \leq A \vee C$. If $\delta=\lambda$, proceed as in (a).

(d) $A$ has less than $\lambda$ proper atoms. Construct an algebra $Q$ with $\lambda$ proper atoms and $Q \equiv 2(\psi)$ similar to $(b)$; then proceed as in (c). This proves that $\left({ }^{*}\right)$ holds for all $\lambda \leq \alpha$.

Now let $\lambda$ be the smallest cardinal such that $|E|=\lambda$ implies $E \neq 2(\psi)$ for all $E \leq D$. Let $A, B \leq D, A \subseteq B$, and $A \equiv B(\psi)$; let $C$ be a complement of $A$ in sub $B$; then $2=A \cap C \equiv_{\psi} B \cap C=C$, and thus, $|C|<\lambda$ by our definition of $\lambda$. This implies $A \equiv B\left(\theta_{\lambda}\right)$.

For the converse, let $A \equiv B\left(\theta_{\lambda}\right), A \subseteq B$, and $C$ a complement of $A$ in sub $B$. Since $A \equiv B\left(\theta_{\lambda}\right)$, we have $|C|<\lambda$, and hence, $C \equiv 2(\psi)$ by 
(*) and our choice of $\lambda$. It follows that $A \equiv_{\psi} A \vee C=B$.

Call a Boolean algebra $D \quad \lambda$-like, if for all $d \in D, D \mid d$ or $D \mid \bar{d}$ has cardinality less than $\lambda$, that is the set $\{d \in D|D| d$ has cardinality less than $\lambda$ \} is a prime ideal of $D$. If, for example, $D$ is the interval algebra of an infinite cardinal $\lambda$, then $D$ is $\lambda$-like. The only countable $\omega$-like algebra is $F C(\omega)$; more generally, it can be shown that an infinite Boolean algebra $D$ is $\omega$-like if and only if $D$ is a finitecofinite algebra.

PROPOSITION 3.9. Let $|D|=\lambda \geq \omega, \lambda$ regular, and $D$ be $\lambda$-like. Then sub $D$ is not simple.

Proof. By 3.6 , it suffices to show that $D$ has the following property :

(*) If $A, B \leq D, u \in D$ such that $|A|=|B|=\lambda$, and $B \leq A(u)$, then there exists a $C \leq D$ with $|C|<\lambda$, such that $(A \cap B) \vee C=B \vee C$.

So, let $A, B$, and $u$ be as described above, and suppose, without loss of generality, that $(\bar{u}]$ has cardinality less than $\lambda$. Using this fact and the regularity of $\lambda$, we may suppose, after a simple thinning process, that there exists a $q \in A$, and, if $B$ is generated by $\left\{b_{i} \mid i<\lambda\right\}$, for each $i<\lambda$ there exists an $a_{i} \in A$ satisfying $b_{i}=a_{i} \circ u+q \circ \bar{u} ;$ furthermore, we may assume that for all $i, j<\lambda$, $a_{i} \circ \bar{u}=a_{j} \circ \bar{u}$. Then, for $i, j<\lambda$.

$$
\begin{aligned}
b_{i} \circ \bar{b}_{j} & =\left(a_{i} \circ u+q \circ \bar{u}\right) \circ\left(\overline{a_{j}}+\bar{u}\right) \circ(\bar{q}+u) \\
& =a_{i} \circ \bar{a}_{j} \circ u \\
& =a_{i} \circ \bar{a}_{j} \in A \text {, since } a_{i} \circ \bar{u}=a_{j} \circ \bar{u} .
\end{aligned}
$$

This in turn implies that also $\overline{b_{i}}+b_{j} \in A$.

If $\left(b_{0}\right]$ has cardinality less than $\lambda$, then so has the set $\left\{b_{0} \circ b_{i} \mid i<\lambda\right\}$, and in this case we set $M=\left(b_{0}\right]$. Since $b_{i}=b_{0} \circ b_{i}+\bar{b}_{0} \circ b_{i}$, and $\bar{b}_{0} \circ b_{i} \in A$, we have $(A \cap B) \vee[M]=B \vee[M]$.

If $\left(\bar{b}_{0}\right]$ has cardinality less than $\lambda$, then so has $\left[b_{0}\right)$; in this case, we set $M=\left[b_{0}\right)$, observing that $b_{i}=\left(b_{0}+b_{i}\right) \circ\left(\bar{b}_{0}+b_{i}\right)$, and $\bar{b}_{0}+b_{i} \in A$. 
As we shall see later, the hypothesis that $\lambda$ is regular, is essential.

Proposition 3.9 also implies a partial answer to problem 29 of [2]:

Call an algebra $D$ almost Jonsson, if for each $B \leq D$ with $|B|=|D|=\lambda$, there exists an $A \leq D$ such that $|A|<\lambda$ and $A \vee B=D$. Call $D$ packed, if $A, B \leq D,|A|=|B|=|D|=\lambda$ imply $|A \cap B|=\lambda$. Note that an almost Jonsson or packed Boolean algebra is $|D|$-like. The question mentioned above asks if there is an almost Jonsson algebra which is not packed, and vice versa.

PROPOSITION 3.10. Let $D$ be an infinite Boolean algebra which is almost Jonsson and has regular cardinality $\lambda$; then $D$ is packed.

Proof. Let $A, B \leq D,|A|=|B|=\lambda ;$ since $D$ is almost Jonsson, there exists a $C \leq D$ with $|C|<\lambda$ which satisfies $A \vee C=D$. Since $B \leq A \vee C$, and $D$ is $\lambda$-like, 3.9 implies the existence of a $Q \leq D$, such that $|Q|<\lambda$, and $(A \cap B) \vee Q=B \vee Q$. Thus, $A \cap B$ must have cardinality $\lambda$.

Next we turn our attention to the interval algebras of well-ordered sets.

If $D$ is the interval algebra of a chain $C$, then each $d \in D^{+}$has a unique representation $d=\left[x_{0}, y_{0}\right) \cup \ldots \cup\left[x_{n}, y_{n}\right)$, where $x_{0}<y_{0}<x_{1}<\ldots<x_{n}<y_{n}$, and possibly $y_{n}=\infty$, that is $\left[x_{n}, y_{n}\right)=\left[x_{n}\right)$. If $d \in D$ has this form, we set $I(d)=\left\{x_{i} \mid i \leq n\right\} \cup\left\{y_{i} \mid i \leq n\right\}$.

PROPOSITION 3.11. Let $\lambda \geq \omega$ be an ordinal and $D$ its interval algebra. Then sub $D$ is not simple if and only if $\lambda$ is a regular cardinat.

Proof. One direction follows immediately from Proposition 3.9, thus, let us suppose that $\lambda$ is not a regular cardinal. In what follows, we shall use the symbols + and $\circ$ both for ordinal addition and multiplication, and for the operations on $D$; the meaning will be clear from the context.

If $\lambda=\beta+\gamma$, with $\beta>\gamma$, then $D \cong I(\beta) \times I(\gamma) \cong I(\gamma) \times I(\beta) \cong I(\gamma+\beta) \cong I(\beta)$, so we can assume that in particular $\lambda$ is a limit ordinal. If $\lambda=\beta \circ n$ for some $n<\omega$, then $D$ 
is isomorphic to the product of $n$ copies of $I(B)$, and it follows from 3.3 that sub $D$ is simple. Thus, let us suppose that $\lambda$ is not of the form $\beta \circ n$, and that of $\lambda=\gamma<\lambda$; then there exists a $\gamma$-termed sequence $\left\{\alpha_{\xi} \mid \xi<\gamma\right\}$ of limit ordinals with supremum $\lambda$, such that $\alpha_{0}=\gamma$, and $\alpha_{\xi} \circ 3<\alpha_{\xi+1}$ for all $\xi<\gamma$.

Our goal is to construct a finite number of subalgebras of $D$, each of which is congruent to 2 , and whose supremum is $D$.

The crucial observation is the following: Let $A_{1} \leq D$ be generated by $\left\{\left[\alpha_{\xi}, \alpha_{\rho}\right) \mid \xi<\rho<\gamma\right\}, A_{2} \leq D$ be generated by $\{[\xi, \rho) \mid \xi<\rho<\gamma\}, A_{3} \leq D$ be generated by $\left\{[\xi, \rho) \cup\left[\alpha_{\xi}, \alpha_{\rho}\right) \mid \xi<\rho<\gamma\right\}$. Then, $A_{1} \cap A_{3}=A_{2} \cap A_{3}=2$, and $A_{1}, A_{2} \leq A_{3}\left(\left[0, \alpha_{0}\right)\right)$; it follows that $A_{1}$ and $A_{2}$ are congruent to 2 , and so is $A=A_{1} \vee A_{2}$. Next, let $B \leq D$ be generated by $\left\{\left[\alpha_{\xi}, \alpha_{\xi} \circ 2\right) \mid \xi<\gamma\right\}$, and $B_{1} \leq D$ be generated by $\left\{[\xi, \xi+1) \cup\left[\alpha_{\xi}, \alpha_{\xi} \circ 2\right) \mid \xi<\gamma\right\}$. Note that $B$ is isomorphic to $F C(\gamma)$; as before, $B \cap B_{1}=2$, and $B \leq B_{1}\left(\left[0, \alpha_{0}\right)\right)$, hence, $B \equiv 2$.

Let $C \leq D$ be generated by $\left\{\left[\alpha+\xi, \alpha_{i}+\rho\right) \mid i<\gamma, \xi<\rho<\alpha_{i}\right\}$, and $R \leq D$ be generated by $\left\{\left[\alpha_{i}+\xi, \alpha_{i}+\rho\right) \cup\left[\alpha_{i} \circ 2+\xi, \alpha_{i} \circ 2+\rho\right) \mid i<\gamma, \xi<\rho<\alpha\right\}$. Let $c \in C^{+}$such that $\lambda \notin I(c)$; then, for each $z \in I(c)$ there exists a $\xi<\gamma$, such that $\alpha_{\xi} \leq z<\alpha_{\xi} \circ 2$; if $r \in R^{+}$such that $\lambda \notin I(r)$, there exists a $z \in I(x)$ and a $\xi<\gamma$ such that $\alpha_{\xi} \circ 2<z<\alpha_{\xi} \circ 3$. It follows that $R \cap C=2$; since $C \leq R \vee B$ and $B \equiv 2$, this implies $C \equiv 2$

For each $i<\gamma$, partition $\left[\alpha_{i+1}, \alpha_{i+1} \circ 2\right)$ into faithfully enumerated subsets $I_{1}^{i}=\left\{m_{\delta} \mid \delta<\alpha_{i+1}\right\}$, and $I_{2}^{i}=\left\{n_{\delta} \mid \delta<\alpha_{i+1}\right\}$, and set

$$
\begin{aligned}
& P_{i}=\left[\begin{array}{llll}
\left\{\left[\alpha_{i} \circ 2+\xi, \alpha_{i} \circ 2+\rho\right)\right. & \cup & \left.\left[m_{\xi}, m_{\rho}\right) \mid \xi<\rho<\alpha_{i+1}\right\}
\end{array}\right] \\
& Q_{i}=\left[\begin{array}{lll}
\left.\left\{\alpha_{i} \circ 2+\xi, \alpha_{i} \circ 2+\rho\right) \cup\left[n_{\xi}, n_{\rho}\right) \mid \xi<\rho<\alpha_{i+1}\right\}
\end{array}\right] .
\end{aligned}
$$

Let $P$ be generated by $\cup\left\{P_{i} \mid i<\gamma\right\}, Q$ be generated by $\cup\left\{Q_{i} \mid i<\gamma\right\}$. 
Similarly as before, it is shown that $P \cap Q=2$. Also,

$$
P \vee A \vee B \vee C=Q \vee A \vee B \vee C=D \text {. }
$$

This implies $P \equiv Q \equiv D$, and it follows from $P \cap Q=2$ that $D \equiv 2$.

Now we can describe the congruences of sub $D$ if $D$ is countable.

PROPOSITION 3.12. Let $D$ be countable; then sub $D$ is not simple if and only if $D$ is isomorphic to $F C(\omega)$.

Proof. If $D \cong F C(\omega)$, then $S u b D$ is not simple by 3.8 . If $D \neq F C(\omega)$, there are two cases:

(a) $D$ contains an infinite free subalgebra: then $S u b D$ is simple by 3.4 .

(b) $D$ does not contain an infinite free subalgebra: then $D$ is superatomic, and it is well known that in this case $D$ is the interval algebra of $\omega^{\beta} \circ n$, where $0<n<\omega$, and $0<\beta<\omega_{1}$. So, sub $D$ is simple by the preceding proposition.

Thus far, all the proper congruences we have exhibited on Sub $D$ were of the form $\theta_{\gamma}$, and in all cases $D$ was $|D|$-like. We would like to conclude this section with an example which shows two things:

1. There exists a Boolean algebra $D$ such that $|D|=\omega_{1}, D$ is not $\omega_{1}$-like, and sub $D$ is not simple.

2. ${ }^{\theta} \omega_{1}$ is not a congruence on sub $D$.

EXAMPLE 3.13. Let $M$ be a subset of the real numbers, such that $M$ has a smallest element and $|M|=\omega_{1}$, and let $E=I(M) \times I(M)$; then $|E|=\omega_{1}, E$ is an interval algebra, and sub $E$ is simple. Now set $D=E \times F C\left(\omega_{1}\right) ;$ then

1. $D$ is not $\omega_{1}$-like;

2. Sub $D$ is sectionally complemented;

3. $\theta_{\omega_{1}}$ is not a congruence on sub $D$;

4. $E$ and $F C\left(\omega_{1}\right)$ have no isomorphic uncountable subalgebras.

For (2), observe that $D$ is a subalgebra of an interval algebra, and to see (3), note that the canonical copy of $E$ in sub $D$ is congruent to 2 , since Sub $E$ is simple. 
Let $u \in D$ such that $D \mid u \cong E$ and $D \mid \bar{u} \cong F C\left(\omega_{1}\right) ;$ let $P, Q \leq D$ be canonical copies of $D \mid u$ and $D \mid \bar{u}$ respectively. Then, $P(u)=(u] \cup[\bar{u})$, and $Q=(\bar{u}] \cup[u)$.

Let $I$ be the ideal of Sub $D$ which is generated by $\{P\} \cup\{S \leq D|| S \mid \leq \omega\}$. Consider the following condition on $I$ :

(*) $(A] \cap(I \vee(B])=((A] \cap I) \vee(A \cap B]$, for all $A, B \leq D$.

(Here, the appearing intervals and $v$ are to be taken in the lattice of ideals of sub $D$. .)

If $I$ satisfies $(*)$, that is if $I$ is standard, then it induces a proper congruence on Sub $D$, see [3], III.3.5. Since $\supseteq$ holds in any lattice, we only have to show $\subseteq$. So, let $A, B, C \leq D, C \leq A$, and $C \leq P(u) \vee T_{1} \vee B$ for some countable $T_{1} \leq D$. We have to show the existence of an $S \leq D$ such that $S \leq A, S \in I$, and $C \leq S \vee(A \cap B)$.

If $A, B$, or $C$ are countable, there is nothing to show, so let us suppose that they are all uncountable.

Let $C_{1}$ be a complement of $C \cap B$ in sub $C$; then $C_{1}^{\prime}=C \cap B \leq A \cap B$.

Let $C_{2}$ be a complement of $C_{1} \cap P(u)$ in sub $C_{1}$; then $C_{2}^{\prime}=C_{1} \cap P(u) \in I$, and $C_{2}^{\prime} \leq A$.

Let $C_{3}=Q(u) \cap C_{2}$ and $T_{2}$ be a complement of $C_{3}$ in sub $C_{2}$. Then, $T_{2} \cap D\left|u=T_{2} \cap D\right| \bar{u}=\{0\}$, which implies that $u$ is independent of $T_{2}$. Assume that $T_{2}$ is uncountable; let $h: T_{2} \rightarrow D \mid u$ be the canonical projection $h(t)=t \circ u$; it is not hard to see that $h$ is an embedding, so $D \mid u$ has a subalgebra isomorphic to $T_{2} ;$ likewise, $D \mid \bar{u}$ has a subalgebra isomorphic to $T_{2} ;$ since $T_{2}$ is uncountable, this contradicts (4).

so, $C_{2}=C_{3} \vee T_{2}$, where $T_{2} \in I$ and $T_{2} \leq A, C_{3} \leq Q(u)$. Since each complement of $C_{3} \cap Q$ in sub $C_{3}$ is finite, we may as well suppose that $C_{3} \leq Q$ 
Let us pause for a moment to recapitulate what we have so far:

(a) $C=C_{1}^{\prime} \vee C_{2}^{\prime} \vee T_{2} \vee C_{3}$;

(b) $C_{1}^{\prime} \leq A \cap B$;

(c) $C_{2}^{\prime} \vee T_{2} \leq A, C_{2}^{\prime} \vee T_{2} \in I$;

(d) $C_{3} \leq Q, C_{3} \cap B=2$.

So we are finished, if we can show that $C_{3}$ is an element of $I$. Now let us look at $B$; let $B_{1}$ be a complement of $B \cap P(u)$ in Sub $B$. If $B_{1}$ is countable, then $B \in I$, and we are done, so, let us suppose that $B_{1}$ is uncountable.

Let $B_{2}=B_{1} \cap Q(u)$ and $B_{2}^{\prime}$ a complement of $B_{2}$ in sub $B_{1}$. Since $\left.\left.B_{2}^{\prime} \cap D\right] U=B_{2}^{\prime} \cap D\right] \bar{u}=\{0\}$, we conclude as in a previous argument that $B_{2}^{\prime}$ is countable. Also as before, we suppose that $B_{2} \leq Q$.

We now have

$$
C_{3} \leq P(u) \vee T_{1} \vee B_{2}^{\prime} \vee B_{2}
$$

Let $R=\left(T_{1} \vee B_{2}^{\prime}\right) \cap Q(u)$ and suppose, without loss of generality, that $u \in R$. Then,

$$
C_{3} \leq P \vee R \vee B_{2}
$$

Now it is not hard to show that $C_{3} \leq R \vee B_{2}$. We also note that $R$ is countable as a subalgebra of $T_{1} \vee B_{2}^{\prime}$.

Our final aim is to show that there is a countable $U \leq Q$ such that $C_{3} \leq U \vee B_{3}$

Let $c \in C_{3}^{+}$, such that, without loss of generality, $c<\bar{u}$, and let $c=r_{0} \circ b_{0}+\ldots+r_{n} \circ b_{n}$ for some $r_{i} \in R, b_{i} \in B_{2}$, and $r_{i} \circ b_{i}>0$ for $i \leq n$.

Let $M$ be the free prime ideal of $D \mid \bar{u}$ generated by the atoms of $D \mid \bar{u}$; then, $Q=M \cup-M$. Since $c \in M$, observe that each $r_{i} \circ b_{i}$ is an element of $M$. Suppose, without loss of generality, that $r_{0}$ is not an 
element of $Q$; then either $r_{0} \leq \bar{u}$, or $r_{0}=u+x$ for some $x<\bar{u}$ with $x \in M$.

(a) $r_{0} \leq \bar{u}$ : then $u+r_{0} \in Q$. Assume that $b_{0} \notin M$, hence, $b_{0}=u+y$ for some $y \notin M$. We now have $r_{0} \circ b_{0}=r_{0} \circ(u+y)$ $=r_{0} \circ y \in M$, which implies $r_{0} \in M$ or $y \in M$, a contradiction. Thus, $b_{0} \in M$, in particular, $b_{0}<\bar{u} ;$ then, $\left(u+r_{0}\right) \circ b_{0}=r_{0} \circ b_{0}$ and $u+r_{0} \in Q$.

(b) $r_{0}=u+x$ for some $x \in M$; then clearly $b_{0} \leq \bar{u}$, $r_{0} \circ b_{0}=x \circ b_{0}$, and $x \in Q$.

If we replace each $r_{i}$ if necessary by one of the elements of $Q$ as described above, and then let $U \leq D$ be generated by these elements and $R \cap Q$, then $U$ is countable, since $R$ is countable, $U$ is a subalgebra of $Q$, and $C_{3} \leq U \vee B_{2}$. Since $C_{3} \cap B_{2}=2$, it follows from 3.7 and 3.8 that $C_{3}$ is countable, hence, $C_{3} \in I$.

\section{Concluding remarks}

Just looking briefly at prime ideals of $\operatorname{sub} D$, we state the following theorem without proof, since it would involve too much new notation and preliminary results which do not seem to be justified.

PROPOSITION 4.1. If $P$ is a prime ideal in sub $D$, then $A \equiv B$ and $A \in P$ imply $B \in P$. It follows that sub $D$ is not simple.

If Sub $D$ is not simple, it need not have a prime ideal. Let $D=F C(\lambda)$, and partition the set of atoms of $D$ into $\left\{x_{i} \mid i<\lambda\right\}$, and $\left\{y_{i} \mid i<\lambda\right\}$; then set $A=\left[\left\{x_{i} \mid i<\lambda\right\}\right], B=\left[\left\{y_{i} \mid i<\lambda\right\}\right]$, and $C=\left[\left\{x_{i}+y_{i} \mid i<\lambda\right\}\right]$. These algebras generate a 0,1 -diamond in sub $D$, so it cannot have a prime ideal. Incidentally, this shows that for no countable $D$ sub $D$ has a prime ideal. Indeed, the only algebra $D$ we know where sub $D$ has a prime ideal is the packed algebra constructed by M. Rubin [4] under $\vartheta_{\omega_{1}}$.

PROBLEM 1. Find an algebra $D$ such that $D$ is not packed, and sub $D$ has a prime ideal. 
The results of the preceding chapter seem to suggest that a nice characterization of those $D$ having sub $D$ (not) simple is hard to come by. All the congruences that we have been able to exhibit on sub $D$ arose from a distributive ideal; this suggests

PROBLEM 2. Find an algebra $D$ and a congruence on sub $D$ which is notinduced by a distributive ideal.

Note that such an algebra cannot have sub $D$ sectionally complemented, in particular, $D$ is not a subalgebra of an interval algebra. Finally, it might be worthy of mention, that the facts that $D \mid d$ has cardinality $\lambda \geq \omega_{1}$ for all $d \in D^{+}$and $\theta_{\lambda}$ is a congruence on sub $D$, imply that $D$ is Bonnet-rigid in the sense of [2].

\section{References}

[1] G. Birkhoff, "Lattice Theory", (American Mathematical Society, Providence, 1948).

[2] E. van Douwen, D. Monk, M. Rubin, "Some questions about Boolean algebras", Algebra Universalis 11 (1980), 220-243.

[3] G. Grätzer, "General Lattice Theory", (Academic Press, New York, Basel, 1978).

[4] M. Rubin, "A Boolean algebra with few subalgebras, interval algebras, and retractiveness", Trans. Amer. Math. Soc. 278 (1983), 65-89.

[5] D. Sachs, "The lattice of subalgebras of a Boolean algebra", Canad. Math. J. 29 (1962), 451-460.

[6] S. Todorčević, "A remark on the lattice of all subalgebras of a Boolean algebra", unpublished.

Department of Mathematics,

University of Brunei Darussalam,

Bandar Seri Begawan,

Brunei. 